\title{
Phase diagram of the extended Hubbard chain with charge-dipole interactions.
}

\author{
M.E. Torio ${ }^{a}$, A.A. Aligia ${ }^{b}$, K. Hallberg ${ }^{b}$ and H.A. Ceccatto ${ }^{a}$. \\ ${ }^{a}$ Instituto de Física Rosario, CONICET-UNR, \\ Bv 27 de Febrero 210 bis, (2000) Rosario, Argentina \\ ${ }^{b}$ Centro Atómico Bariloche and Instituto Balseiro, \\ Comisión Nacional de Energía Atómica, \\ 8400 Bariloche, Argentina.
}

\begin{abstract}
We consider a modified extended Hubbard model (EHM) which, in addition to the on-site repulsion $U$ and nearest-neighbor repulsion $V$, includes polarization effects in second-order perturbation theory. The model is equivalent to an EHM with renormalized $U$ plus a next-nearestneighbor repulsion term. Using a method based on topological quantum numbers (charge and spin Berry phases), we generalize to finite hopping $t$ the quantum phase diagram in one dimension constructed by van den Brink et al. (Phys. Rev. Lett. 75, 4658 (1995)). At hopping $t=0$ there are two charge density-wave phases, one spin density-wave phase and one intermediate phase with charge and spin ordering, depending on the parameter values. At $t \neq 0$ the nature of each phase is confirmed by studying correlation functions. However, in addition to the strong-coupling phases, a small region with bond ordering appears. The region occupied by the intermediate phase first increases and then decreases with increasing $t$, until it finally disappears for $t$ of the order but larger than $U$. For small $t$, the topological transitions agree with the results of second order perturbation theory.
\end{abstract}

Pacs Numbers: buscar, 71.10.Fd

\section{INTRODUCTION}

Atomic polarizabilities are very important in solid state physics, since they contribute to the screening of the effective on-site repulsion. For example, from atomic data the bare on-site Coulomb energy for $\mathrm{Cu}^{+2}$ is $U_{0}=E\left(\mathrm{Cu}^{+3}\right)+E\left(\mathrm{Cu}^{+}\right)-2 E\left(\mathrm{Cu}^{+2}\right) \sim 17 \mathrm{eV}$, while constrained density functional calculations for $\mathrm{La}_{2} \mathrm{CuO}_{4}$ give $U \sim 10 \mathrm{eV}$ 目. In the case of $\mathrm{BaBiO}_{3}$, while $U_{0}\left(\mathrm{Bi}^{+4}\right) \sim 11 \mathrm{eV}$, it has peen suggested that the effective $U$ becomes negativel. Interesting effects of the polarizability also appear in the physics of small clusters. In particular, while in neutral clusters of a few atoms of $\mathrm{Hg}$ the only relevant interatomic interactions are van der Waals forces (originated by fluctuations of the polarization), a transition to covalent and finally to metallic honding takes place as the size of the cluster increases $\mathrm{E}$ - A. A very good fit of the observed ionization potential as a function of cluster size has been obtained using a theory which includes charge-dipole and dipole-dipole interactions in a two band model (representing the $6 \mathrm{~s}$ and $5 \mathrm{p}$ electrons of $\mathrm{Hg}$ ).

Recently, a simple one-band model including screening effects has been proposed and studied.9.10 The main simplifying assumption is that the electronic transitions responsible for the screening do not involve the valence electrons (only transitions from one deep level to another one well above the Fermi energy are considered). Furthermore, only nearest-neighbor charge-dipole interactions are included. These facts renders the model more amenable to many-body treatments, and interesting conclusions have been extracted. In particular, in one dimension (1D), in addition to the usual charge density wave (CDW1) and spin density wave (SDW) phases of the extended Hubbard chain, two newphases appear in the ground state for hopping $t=0$. Id: a charge density wave with a unit cell of four lattice parameters (CDW2), and a combined spin and charge density wave with a unit cell of six lattice parameters called intermediate (I) phase. The 1D phase diagram for $t=0$ was constructed, and the energy gap, static correlation functions and oneparticle excitation spectra have cally for a few cases with $t \neq 0.0 .10$ However, the phase diagram for $t \neq 0$ has not been investigated yet.

The main difficulty in obtaining the phase diagram for $t \neq 0$ is that, for finite systems, the conventional order parameters are smooth functions of the parameters of the model, and the values at which the transitions take place in the thermodynamic limit cannot be determined precisely. However, recently it has been shown that in certain cases, including the usual extended Hubbard model with repulsive interactions 1 , the thermodynamic phases are characterized by different topological numbers (determined by charge and spin Berry phases), and these jump suddenly for any system size 1113 Extrapolating the position of this jump to the thermodynamic limit, a very accurate determination of phase boundaries was obtained, as established by comparison with available exact results in the strong-coupling limit and field-theory ones in the weak-coupling case 12,4 In 1D, the method of the Berry phases is related to the method of crossings of excitacion levels based on the conformal field theory with renormalization group analysis.15. 16 This method (abbreviated as level-crossing method in the rest of the paper) is not expected to work in the strong coupling limit.

In this work we use the method of Berry phases to calculate the phase diagram of the model for $t \neq 0$. 
In particular, we show that in this case all the thermodynamic phases can be characterized by the topological numbers, including also a new phase with bond ordering previously suggested in the literature.16 The nature of each phase is confirmed by calculating different correlation functions. In Section II we describe the model and the strong-coupling thermodynamic phases. Section III contains a brief description of the calculation of the topological numbers and the values they take for the $t=0$ phases. In Section IV we present the quantum phase diagram for several values of $U_{0} / t$, compare it with the results of perturbation theory in $t$, and discuss the behavior of the correlation functions. In Section $\mathrm{V}$ we summarize our results and discuss the extent and limitations of the method of the topological transitions and that of the level crossings.

\section{MODEL AND $T=0$ GROUND-STATE PHASES}

The model is defined by the Hamiltonian

$$
H=H_{0}+H_{c d}
$$

where $H_{0}$ is the usual extended Hubbard chain:

$$
\begin{aligned}
H= & -t \sum_{i \sigma}\left(c_{i+1 \sigma}^{\dagger} c_{i \sigma}+\text { H.c. }\right)+U_{0} \sum_{i} n_{i \uparrow} n_{i \downarrow} \\
& +V \sum_{i} n_{i} n_{i+1}
\end{aligned}
$$

and $H_{c d}$ describes charge-dipole interactions (dipoledipole interactions are neglected):

$$
H_{c d}=-\sum_{i} \mathbf{p}_{i} \cdot \mathbf{E}_{i}
$$

Here $\mathbf{p}_{i}$ and $\mathbf{E}_{i}$ are the dipole moment operator and electric field at site $i$ respectively. Treating this term in second-order perturbation theory 10 it takes the form $-\frac{1}{2} \sum_{i} \alpha_{i} E_{i}^{2}$, where $\alpha_{i}$ is the polarizability at site $i$. Considering only the contribution to the electric field coming from nearest-neighbor atoms, and assuming that $\alpha_{i}$ is independent of the site and its occupancy, one can write:

$$
H_{c d}=-P \sum_{i}\left(n_{i+1}-n_{i-1}\right)^{2}
$$

where $P=\alpha e^{2} /\left(2 a^{4}\right)$ and $a$ is the lattice parameter. Expanding $H_{c d}$, the total Hamiltonian can be cast into the form:

$$
\begin{aligned}
H= & -t \sum_{i \sigma}\left(c_{i+1 \sigma}^{\dagger} c_{i \sigma}+\text { H.c. }\right)+\left(U_{0}-4 P\right) \sum_{i} n_{i \uparrow} n_{i \downarrow} \\
& +V \sum_{i} n_{i} n_{i+1}+2 P \sum_{i} n_{i} n_{i+2}-2 P \sum_{i} n_{i} .
\end{aligned}
$$

The last term merely renormalizes the chemical potential. As stated before 10 , within the above mentioned approximations $H_{c d}$ reduces the on-site interaction and generates a next-nearest-neighbor repulsion.
We are interested in the half-filled case of one particle per site. For $t=0$ the model can be solved exactly 10 , and at this filling four different phases appear in the ground state depending on the parameter values. We use the notation $\bigcirc, \bullet, \uparrow$, and $\downarrow$, to represent empty, doubly occupied, and singly occupied sites with spin up and down respectively. If $V$ is much larger than the other interactions, nearest-neighbor occupancy must be avoided and the ground state can be represented as $(\ldots \bigcirc \bullet \bigcirc \bullet \bigcirc \bullet \bigcirc \bullet \ldots)$. This state is denoted as charge density wave 1 (CDW1). If $U_{0}$ is the dominant interaction all sites are singly occupied, and an infinitesimally small $t$ is enough to introduce antiferromagnetic correlations between nearest-neighbors. Thus, we represent this spin density wave (SDW) as (... $\downarrow \uparrow \downarrow \uparrow \downarrow \ldots)$, although there is no true spin longrange order in one dimension. If $P$ is very large, occupancy at next nearest neighbors is avoided and the ground state $(\mathrm{CDW} 2)$ is $(\ldots \bigcirc \bigcirc \bullet \bullet \bigcirc \bigcirc \bullet \bullet .$.$) . Finally,$ for intermediate values of the parameters (for example $\left.V=0.5 U_{0}, P=0.15 U_{0}\right)$ there is an intermediate (I) phase, the unit cell of which can be represented as $\bullet \uparrow$ $\bigcirc \bullet \downarrow \bigcirc$.

\section{BERRY PHASES}

We have calculated the ground state $\left|g\left(\Phi_{\uparrow}, \Phi_{\downarrow}\right)\right\rangle$ of the model in rings of different lengths $L$, threaded by fluxes $\Phi_{\sigma}$ for spin $\sigma$ (the hopping for each spin is affected a factor $e^{i \Phi_{\sigma} / L}$ as described in detail elsewhere 11 13). We call charge (spin) Berry phase $\gamma_{c}$ $\left(\gamma_{s}\right)$, the phase captured by the ground state when it is followed adiabatically in the cycle $0 \leq \Phi \leq 2 \pi$, keeping $\Phi_{\uparrow}=\Phi_{\downarrow}=\Phi\left(\Phi_{\uparrow}=-\Phi_{\downarrow}=\Phi\right)$. Discretizing the interval $0<\Phi<2 \pi$ into $N$ points $\Phi_{i}=2 \pi i / N$, the Berry phases are calculated numerically by the gaugeinvariant expression 11 :

$$
\begin{aligned}
\gamma_{c, s}= & -\lim _{N \rightarrow \infty}\left\{\operatorname { I m } \left[\operatorname { l n } \left(\Pi_{i=0}^{N-2}\left\langle g\left(\Phi_{i}, \pm \Phi_{i}\right) \mid g\left(\Phi_{i+1}, \pm \Phi_{i+1}\right)\right\rangle\right.\right.\right. \\
& \left.\left.\left.\left\langle g\left(\Phi_{N-1}, \pm \Phi_{N-1}\right) \mid g(2 \pi)\right\rangle\right)\right]\right\}
\end{aligned}
$$

where $|g(2 \pi)\rangle$ represents $|g(2 \pi, \pm 2 \pi)\rangle$ obtained directly from $|g(0,0)\rangle$ :

$$
|g(2 \pi)\rangle=\exp \left[i \frac{2 \pi}{L} \sum_{j} j\left(n_{j \uparrow} \pm n_{j \downarrow}\right)\right]|g(0,0)\rangle .
$$

An important property of the charge Berry phase is that if the system is modified by some perturbation, the change in the polarization $P_{\uparrow}+P_{\text {is }}$ is proportional to the corresponding change in $\gamma_{c} .17$ Here $P_{\sigma}$ is the contribution of electrons with spin $\sigma$ to the polarization of the system. Similarly, changes in $\gamma_{s}$ are related to changes in the difference $P_{\uparrow}-P_{\downarrow}$ betyeen the electric polarizabilities for spin up and down 13 .

$$
\Delta P_{\uparrow} \pm \Delta P_{\downarrow}=e \Delta \gamma_{c, s} / 2 \pi[\bmod (e)]
$$

A crucial property for our purposes is that, in systems with inversion symmetry like ours, $\gamma_{c}$ and $\gamma_{s}$ can only be either 0 or $\pi[\bmod (2 \pi)]$ (the argument of the 
logarithm in Eq. (6) turns into its complex conjugate under inversion). Then, the Berry phase vector $\gamma=\left(\gamma_{c}, \gamma_{s}\right)$ cannot vary continuously, and a jump in $\gamma$ corresponds to a transition in at least one of the topological numbers $\gamma_{c} / \pi, \gamma_{s} / \pi$. Our main hypothesis is that the topological transitions correspond to phase transitions in the thermodynamic limit. This has been confirmed by different numerical and analytical stuedies in the Hubbard model with correlated hopping. 12.14 In the present case, we check this by comparing different correlation functions (see next section). In addition, it has been shown that the opening of a spin gap in a Luttinger liquid phase of a spin rotational invariant model is accompanied by a topological transition in $\gamma_{s} 13$.

Let us discuss the values of $\gamma$ for the four possible ground states of Eq.(1) in the half-filled system with $t=0$, discussed at the end of the previous section. For $t=0$, we can choose a gauge in which all scalar products in Eq. (6) are equal to 1, except eventually the last one. Then the Berry phases are defined by the argument of the exponential in Eq. (7). If the ground state is the state $(\ldots \bigcirc \bullet \bigcirc \bullet \bigcirc \bullet \bigcirc \bullet \ldots)$ of the CDW1, then this argument is clearly 0 for $\gamma_{s}$, while for $\gamma_{c}$ it is equal to $i \frac{2 \pi}{L} \sum_{j} j\left(n_{j \uparrow}+n_{j \downarrow}\right)=i \frac{2 \pi}{L} \sum_{m=1}^{L / 2} 4 m=$ $i 2 \pi(L+2) \equiv 0[\bmod (2 \pi i)]$. The same result is obtained for the other CDW1 state $[\ldots \bullet \bigcirc \bullet \bigcirc \bullet \bigcirc \bullet \bigcirc \ldots]$, so $\gamma(\mathrm{CDW} 1)=(0,0)$. One can construct a Néel state (representing the SDW) displacing half of the electrons (for example those with spin up) one lattice parameter either to the left or to the right. Then, from Eqs. (8) one has $\gamma(\mathrm{SDW})=(\pi, \pi)$. This result can also be easily obtained calculating the expepent of (7), and is not altered by spin fluctuations 11,22 For the CDW2 with order $(\ldots \bigcirc \bigcirc \bullet \bullet \bigcirc \bigcirc \bullet \bullet \ldots)$ clearly $\gamma_{s}=0$, because the spin at each site is compensated. For $\gamma_{c}$ one has $\frac{2 \pi}{L} \sum_{j} j\left(n_{j \uparrow}+n_{j \downarrow}\right)=\frac{2 \pi}{L} \sum_{m=1}^{L / 4}(16 m-2)=2 \pi(L+$ $4-1 / 2) \equiv \pi[\bmod (2 \pi)]$. Then, $\gamma(\operatorname{CDW} 2)=(\pi, 0)$. While the phases above have been calculated for $t=$ 0 , it is clear that by continuity each thermodynamic phase retain the same value of $\gamma$ unless a topological transition takes place.

The vector $\gamma$ in the I phase cannot be calculated as easily as above for $t=0$. The reason is that the state $(\ldots \bullet \uparrow \bigcirc \bullet \downarrow \bigcirc \ldots)$ does not have a definite parity under inversion and other states related with it by inversion have different exponents of Eq. (7). For $t \neq 0$ the ground state should have a well defined parity, and $\gamma$ depends on the particular linear combination of the above mentioned states which constitutes the ground state. Numerically we found $\gamma(\mathrm{I})=(0, \pi)$. This is a fortunate fact, since then at $t=0$ the four thermodynamic phases are characterized by all the possible values of $\gamma$. Thus, the corresponding phase boundaries can be determined from topological transitions. Notice, however, that this does not rule out the possibility that at $t \neq 0$ new phases having the same $\gamma$ values could appear. We will show in the next section that this is in fact what happens. To summarize and for future use, the Berry phase vectors for each of the strong-coupling phases are $\gamma(\mathrm{CDW} 1)=(0,0)$,
$\gamma(\mathrm{SDW})=(\pi, \pi), \gamma(\mathrm{CDW} 2)=(\pi, 0)$, and $\gamma(\mathrm{I})=(0, \pi)$.

\section{NUMERICAL RESULTS}

In this section we present the ground-state phase diagram of model (11) for $t \neq 0$. As discussed in the previous section, it has been obtained by studying the behavior of the Berry phases in rings of $L=8$ and 12 sites. Notice however that the order corresponding to the I phase cannot be accommodated in rings of 8 sites because of its 6 -site unit cell. In Fig. 1 we show the phase diagram for $U_{0} / t=10,3$ and 1 , which correspond to the strong to moderate coupling regimes. As can be seen in Fig. 1a, for $U_{0} / t=10$ the results are similar to the strong limit case $(t=0)$. Moreover, there are no sensible differences between the values obtained in rings of 8 and 12 sites, which indicates that they are representative of the thermodynamic limit results. Fig. 1b shows that already for $U_{0} / t=3$ the behavior departs from the one corresponding to the strong coupling limit; in particular, there are stronger finite-size effects in the transition lines CDW1-I and CDW2-I, especially when the transition line is determined through the jump in the spin Berry phase. The line CDW2-I is better determined by the changes in the charge Berry phase, which shows no major finitesize effects. We expect that, for this line, going to larger systems the jumps in the spin Berry phase will coincide with those of the charge Berry phase.

Note that with a small increase in $t$ from the strong coupling limit, the I phase first increases with respect to the CDW1 and CDW2 phases. This can be understood from perturbation theory in $t$. The energies of the different phases calculated up to second order in $t$ are the following:

$$
\begin{gathered}
E(\mathrm{SDW})=N\left(V+2 P-\frac{4 t^{2} \ln 2}{U_{0}-4 P-V}\right), \\
E(\mathrm{CDW} 1)=N\left(\frac{U_{0}}{2}+2 P-\frac{2 t^{2}}{3 V-U_{0}}\right), \\
E(\mathrm{CDW} 2)=N\left(\frac{U_{0}}{2}-2 P+V-\frac{t^{2}}{12 P-V-U_{0}}\right), \\
E(\mathrm{I})=\frac{N}{3}\left[U_{0}+2 V-t^{2}\left(\frac{1}{P}+\frac{2}{8 P+V-U_{0}}\right)\right] .
\end{gathered}
$$

The phase diagram that results from these energies is represented in Fig. 2. For $U_{0} / t=3$, the second order expression for $E(\mathrm{SDW})$ is no more valid (it gives a very low energy). Thus, we have not drawn the boundaries of the SDW phase for this value of $U_{0} / t$. Instead, the other boundaries of the I phase, and in general all boundaries for $U_{0} / t=10$ (except when some denominator becomes of the order of $t$ ), agree very well with the topological transitions represented in Fig. 1. 

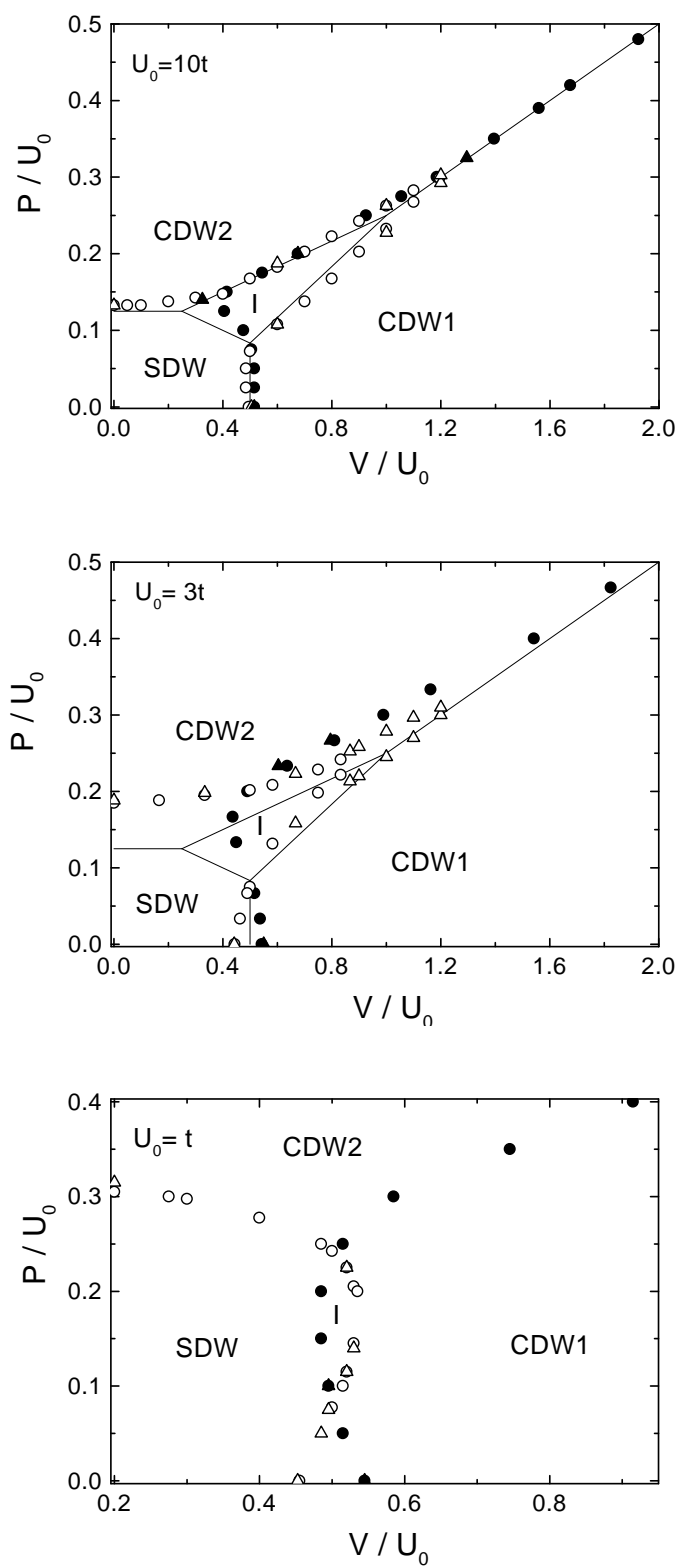

FIG. 1. Quantum phase diagram of model (11). a) $U_{0} / t=10$; b) $U_{0} / t=3$; c) $U_{0} / t=1$. Solid dots and triangles correspond to jumps in the charge Berry phase obtained by considering rings with $L=8$ and 12 sites respectively; open dots and triangles indicate jumps in the spin Berry phase for systems of the same length. The thin lines correspond to the $t=0$ phase diagram.

From Eqs. (9) and Fig. 2, we see that near the points where the CDW1, CDW2 and I phases meet for $t=0$ the stability of the I phase increases with $t$. This is due to the fact that the electrons of the doubly occupied sites of the I phase can jump to the neighboring singly occupied sites with small energy cost in $P$, and without increasing on-site and nearest-neighbor repulsion energies. As a consequence, for small $t \neq 0$, the electrons in the I phase become more mobile and gain more kinetic energy than in the other two phases.

On the other hand, for $U_{0} / t=3$ the topological transitions seem to indicate that a new phase appears between the SDW and CDW1 phases (see Fig. 1). This was only slightly visible for $U_{0} / t=10$ and is out of the reach of the perturbative calculations. We wave studied this possibility in more detail by considering the scaling of critical points on the axis $P=0$ shown in Fig. 3. From this figure we see that the jumps in the spin and charge Berry phases will not coincide even in the thermodynamic limit; this fact is pointing to the emergency of a new order termed bond ordering wave (BOW), as suggested in a recent study of the phase diagram of the EHM.16

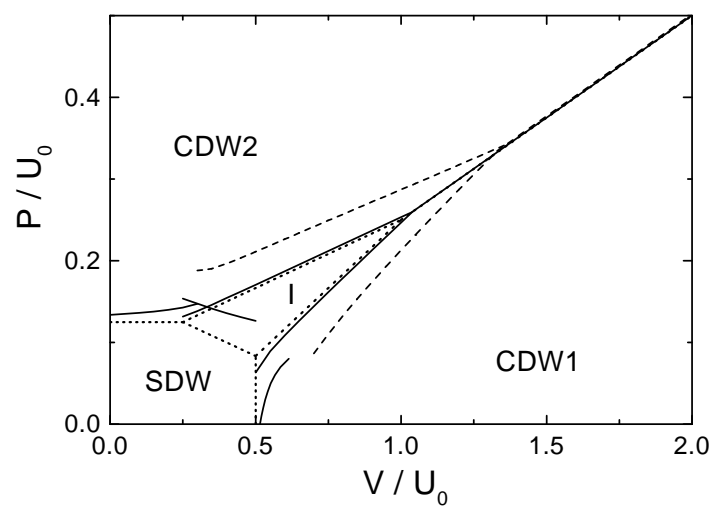

FIG. 2. Phase boundaries obtained by perturbation theory in $t$. Full line $U_{0} / t=10$, dashed line $U_{0} / t=3$. The dotted line is the known result for $t=0.9$.

For $U_{0} / t=1$ (see Fig. 1c) the phase diagram looks quite different to the strong coupling case, with the I phase shrinking from a triangular-like shape to a smaller biconvex region. Furthermore, the BOW phase remains present without much change. Notice that using bosonization (valid in the extreme weakcoupling regime $t \rightarrow \infty$ ), the BOW phase is absent for $P=0,14,18$ and the I and CDW2 phases should also disappear since the expected instabilities have wave vector $2 k_{F}=\pi$.

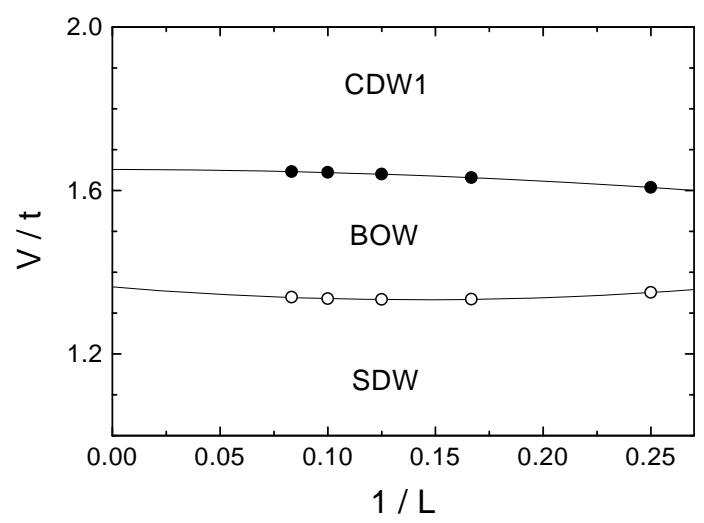

FIG. 3. Values of $V / t$ at which the charge (solid dots) and spin (open dots) Berry phases jump on the $P=0$ axis, as a function of the ring length $L$. We have taken $U_{0} / t=3$.

In order to have a better characterization of the order present in the different phases, we have also computed static correlation functions along the $P=0$ axis from $V / t=1$ to $V / t=2$, that is, traversing across the SDW, BOW and CDW1 phases. Fig. 4 shows the spin and charge correlation func- 
tions at the largest distance $L / 2=5$ for a 10site chain, and also the correlation function corresponding to the expected order in the BOW phase, $C_{\mathrm{BOW}}(L / 2)=\left\langle\left[O_{\mathrm{B}}(L / 2)-\overline{O_{\mathrm{B}}}\right]\left[O_{\mathrm{B}}(0)-\overline{O_{\mathrm{B}}}\right]\right\rangle$, with $O_{\mathrm{B}}(j)=(-)^{j} \sum_{\sigma}\left(c_{\sigma, j+1}^{\dagger} c_{\sigma, j}+\right.$ H.c. $)$. The vertical dotted lines correspond to the transition points extrapolated in Fig. 2, and we can see the expected behavior for these three functions along the chosen path.

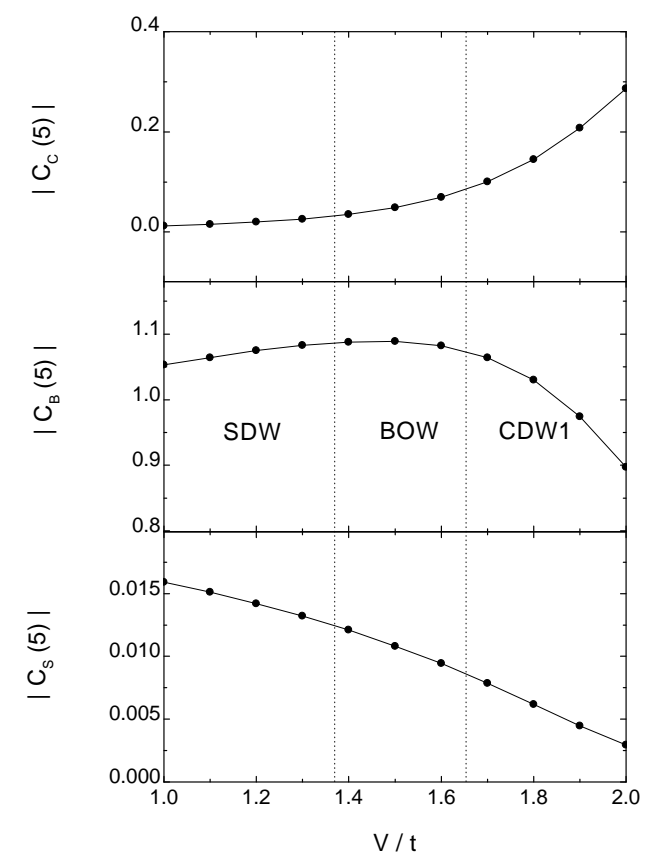

FIG. 4. Static charge $\left(C_{\mathrm{C}}\right)$, BOW $\left(C_{\mathrm{B}}\right)$ and spin $\left(C_{\mathrm{S}}\right.$ correlation functions at the largest distance $L / 2=5$ for a 10-site ring. The dotted vertical lines indicate the extrapolated points in Fig. 3.

Furthermore, in Fig. 5 we plot the fluctuations of the three order parameters [for example $\chi_{\mathrm{BOW}}=$ $\left.\frac{1}{L^{2}} \sum_{i j} e^{i q\left(R_{i}-R_{j}\right)}\left\langle O_{\mathrm{B}}(i) O_{\mathrm{B}}(j)\right\rangle\right]$ at their peak values (corresponding to $k=\pi$ ), as a function of the ring length and for $V / t=1.5$, that is, inside the BOW phase. Both the spin and charge fluctuations diminish with increasing lengths, while the BOW fluctuations increase and most likely dominate in the thermodynamic limit. The same analysis in the SDW and CDW1 phases is not so conclusive because the BOW fluctuations do not clearly decay when increasing the size of the system; however, since the orders present for small and large values of $V / t$ are not a matter of discussion, this must be only due to the small size of the systems considered.

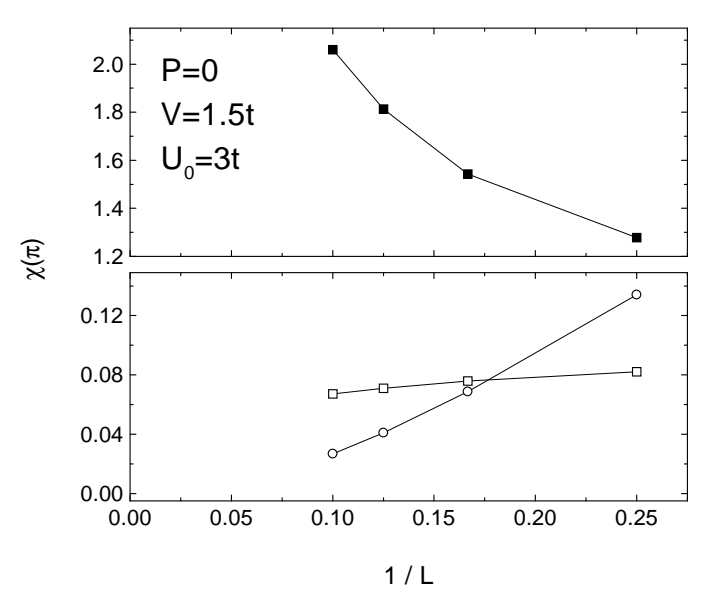

FIG. 5. Charge (open dots), spin (open squares) and BOW (solid dots) order-parameter fluctuations at $k=\pi$ as a function of the ring length $L$. We have considered the parameters values $P=0, V / t=1.5$ and $U_{0} / t=3$, which correspond to a point well inside the BOW phase.

\section{DISCUSSION}

We have studied the effect of electronic polarization $P$ on the phase diagram of the 1D extended Hubbard model. Following Refs. 1 .10, we have neglected the screening effect of valence electrons, although it was important to explain experiments in $\mathrm{Hg}$ clusters $\mathrm{d}$. At $P=0$, for a bipartite lattice in any dimension two phases are expected: a SDW for large on-site Coulomb repulsion $U_{0}$ (without true long-range order in $1 \mathrm{D}$ ), and a CDW1 for large mearest-neighbor repulsion $V$. In $1 D$ the results of Ref 16 and ours support the existence of a narrow region of long-range bond ordering between the other two phases, if the hopping $t \neq 0$. Previous studies in 1D9, 10 have shown that for small $t$ and sufficiently large $P$, two new phases appear: CDW2 and I, with unit cells containing four and six lattice parameters respectively, as described in section II.

For $t=0$ it is clear (see section II) that a transition from one phase to the other is accompanied by charge and /or spin transport. Since the charge Berry mhase $\gamma_{c}$ is a measure of the polarization of the system, 17 and similarly a jump in the spin Berry phase $\gamma_{s}$ indicates a jump in the difference in polarization for spin up and down, 13 it seems appropriate to determine the phase diagram frem the jumps in the topological numbers $\gamma_{c} / \pi, \gamma_{s} / \pi .12$ Using this method, we find that for fixed $U_{0}, V$ and $P$, the regions occupied by the CDW2 and particularly the I phase, are reduced for sufficiently large $t$. This result is expected on general grounds, since only instabilities with wave vector $\pi$ are expected in the weak coupling limit $(t \rightarrow \infty)$, which shoulf well described by continuum limit field theory. 14.18 .19 For small $t$ (up to $U_{0} / 3$ ) the region occupied by the I phase first increases with $t$, and this effect can be understood using second-order perturbation theory in $t$.

Although in the present case we succeded in determining all phase boundaries from the jumps in the 
topological numbers $\gamma_{c} / \pi, \gamma_{s} / \pi$, there are some cases in which the thermodynamic phases are characterized by the same topological numbers, and the phase transition cannot be detected. In other cases some topological transitions do not have an obvious physical meaning.13,20 An example of the former is the transition between SDW and triplet superconductivity which takes place for positive $U$ and negative $V$ in the extended Hubbard model in $1 \mathrm{D}$, which is however well determined by the level-crossing method 16 This method, as used in Refs.15,16, is limited to 1D and is based on the fact that for critical systems, due to conformal invariance, the exponent of the distance dependence of different correlation functions is given by the corresponding excitation energies 15 For systems at (or slightly out of) criticality, described by the sine-Gordon model at low energies, renormalization arguments were used and logarithmic finite-size corrections can be eliminated mingappropriate combination of excitation energies. 152122 This method cannot be applied to phases which are out of the reach of the continuum-limit field theory like the CDW2 and I phases, or when there is phase separation. Instead, in the strong-coupling limit $(t \rightarrow 0)$ it is usually very simple to calculate the Berry phases, even in some phase separated states 1320 (it reduces to a simple counting argument, as shown in Section II), and the method of the topological numbers is very precise. It is interesting to note that, in spite of their different meaning and expected limitations, both methods give the same results for transitions among the SDW, CDW1 and BOW phases. The method of Berry phases has also been applied to $2 \mathrm{D}$ transitions 17 .

\section{ACKNOWLEDGMENTS}

One of us (AAA.) thanks J. Lorenzana for useful discussions. MET and HAC. acknowledge support from CONICET. AAA. is partialy supported by CONICET. This work was sponsored by PICTs 03-00121-02153 and 03-03833 of ANPCyT and PIP 4952/96 of CONICET.

${ }^{1}$ J.F. Annet, R.M. Martin, A.K. Mc Mahan and S. Satpathy, Phys. Rev. B 40, 2620 (1989).

${ }^{2}$ M.S. Hybertsen, E.B. Stechel, M. Schlüter, and D.R. Jeninson, Phys. Rev. B 41, 11068 (1990); references therein.

${ }^{3}$ J.B. Grant and A.K. Mc Mahan, Phys. Rev. Lett. 66, 488 (1991).

${ }^{4}$ C.M. Varma, Phys. Rev. Lett. 61, 2713 (1988).

${ }^{5}$ K. Rademann, B. Kaiser, U. Even, and F. Hensel, Phys. Rev. Lett. 59, 2319 (1987).

${ }^{6}$ C. Bréchignac, M. Broyer, Ph. Cahuzac, G. Delacretaz, P. Labastie, J.P. Wolf, and L. Wüste, Phys. Rev. Lett. 60, 275 (1988).
${ }^{7}$ M.E. García, G. Pastor, and K.H. Bennemann, Phys. Rev. Lett. 67, 1142 (1991).

${ }^{8}$ A.A. Aligia, M.E. García, and K.H. Bennemann, Europhys. Lett. 21, 177 (1993).

9 J. van den Brink, M.B.J. Meinders, J. Lorenzana, R. Eder, and G.A. Sawatzky, Phys. Rev. Lett. 75, 4658 (1995).

${ }^{10}$ M.B.J. Meinders, J. van den Brink, J. Lorenzana, and G.A. Sawatzky, Phys. Rev. B 52, 2484 (1995).

11 A.A. Aligia and E.R. Gagliano, Physica C 304, 29 (1998).

12 A.A. Aligia, K. Hallberg, C.D. Batista, and G. Ortiz; Phys. Rev. B 61, 7883 (2000); J. Low Temp. Phys. 117, 1747 (1999).

13 A.A. Aligia, Europhys. Lett. 45, 411 (1999).

14 A.A. Aligia and L. Arrachea, Phys. Rev. B 60, 15332 (1999); references therein.

${ }^{15}$ K. Nomura and K. Okamoto, J. Phys. A: Math. Gen. 27, 5773 (1994).

${ }^{16}$ M. Nakamura, J. Phys. Soc. Jpn. 68, 3123 (1999); Phys. Rev. B 61, 16377 (2000), references therein.

17 G. Ortiz, P. Ordejón, R. Martin, and G. Chiappe, Phys. Rev. B 54, 13515 (1996).

18 J. Japaridze, Phys. Lett. A 201, 239 (1995).

19 J. Voit, Rep. Prog. Phys. 58, 977 (1995).

${ }^{20}$ A.A. Aligia and G. Ortiz, Phys. Rev. Lett. 82, 2560 (1999).

${ }^{21}$ I. Affleck, D. Gepner, H.J. Schulz and T. Ziman, J. Phys. A: Math. Gen. 22, 511 (1989).

${ }^{22}$ K.Hallberg, X.Wang, P.Hirsch and A.Moreo, Phys. Rev. Lett. 76, 4955 (1996). 